\title{
Analysis of the data of IMF Bz and AE index for the period 1999-2018
}

\author{
Yuryi Polozov* \\ Institute of Cosmophysical Research and Radio Wave Propagation FEB RAS, 684034, Kamchatskiy \\ kray, Paratunka, Russian Federation
}

\begin{abstract}
Solar-terrestrial relations are a part of space weather and they make significant impact on technosphere functioning. Interplanetary Magnetic Field (IMF) parameters measured at Lagrange point L1 show the beginning of possible changes in geophysical fields. The paper analyzes statistically IMF Bz component (shows the level of impact on the Earth magnetic field) and $\mathrm{AE}$ index (used to estimate the energy contribution into magnetosphere and ionosphere). Using the apparatus of regression analysis and wavelet transform, time series were estimated. Regression models of IMF parameters and AE index were developed for the period 1999-2018.
\end{abstract}

\section{Introduction}

Mechanism of solar-terrestrial relations provides energy transfer from interplanetary space to geospheric shells that significantly affects technosphere and human life. The important mechanism of energy transfer from the Sun to the Earth's magnetosphere is the magnetic reconnection [1]. It takes place if interplanetary magnetic field (IMF) lines are directed southward and IMF and geomagnetic field are connected in the magnetopause region. After this connection, IMF lines move to the magnetosphere tail [1]. That provides solar wind plasma transfer into the inner magnetosphere [2]. Significant energy is transferred during magnetic storms and sub-storms occurring at the times of coronal mass ejections (CME), corotation interaction regions (CIR) and high speed solar wind streams (HSS) which carry Alfven waves $[3,4]$. Solar wind energy volumes entering the inner magnetosphere depend on IMF line directions. The time of IMF line turn to the south can be detected on the basis of IMF Bz-component. This component is often used to analyze and to forecast geo-effective events $[4-6]$.

To calculate the energy penetrated into the inner magnetosphere and ionosphere, geomagnetic activity auroral index (AE) is generally used $[4,7]$. AE-index characterizes energy change in ionospheric electrojets located at the height of about $100 \mathrm{~km}[8]$. AE index

\footnotetext{
* Corresponding author: up_agent@mail.ru
} 
increases in the result of CME events [9], Alfven oscillation impact when meeting HSS [3, 4, 10], CIR effect [11]. Based on the abovesaid, IMF Bz is generally used to estimate AE index (for example, [4-6]). IMF Bz parameter is measured at L1 point and it shows the changes 20-30 minutes before the changes in AE index during geo-effective events [3,4].

In the paper we used an apparatus of correlation analysis and regressive models to estimate the relation between IMF Bz parameters and AE index over the period 1999-2018. Based on the apparatus of continuous wavelet transforms, IMF $\mathrm{Bz}$ parameters of different frequency were detected. Intensity measure was introduced and regressive models were developed for the frequency ranges. On the basis of the obtained models, we estimated the impact of different frequency ranges of IMF Bz on AE-index. Statistical calculations of the models showed their significance and the fact of considerable influence of IMF $\mathrm{Bz}$ parameters on auroral electrojet state.

\section{Methodology}

In the paper we used the IMF Bz data (GSM coordinates) obtained on board ACE (Advanced Composition Explorer) spacecraft. The ACE spacecraft is located at a libration point L1. The data sampling was 1 hour. The hourly data were chosen because they allow us to reduce the lag effect when constructing correlations and have less gaps compared to 1-minute data. Gaps in the data were filled in by the nearest neighbor method [12]. If there were gaps with the duration of 8 hours and more during a day or a continuous gap of 4 hours and more, the event was removed from the analysis. The data are available at the site https://omniweb.gsfc.nasa.gov. To estimate the energy in electrojet auroral region, AE index was used. Data sampling was 1 hour. The data were obtained from the site http://wdc.kugi.kyoto-u.ac.jp. Data for IMF Bz and AE were used for the period 1999-2018 (covering the 23-24 solar cycles).

IMF $\mathrm{Bz}$ data were processed by the apparatus of continuous wavelet-transform [13]:

$$
\left(W_{\Psi} f_{b, a}\right):=|a|^{-\frac{1}{2}} \int_{-\infty}^{+\infty} f(t) \Psi\left(\frac{t-b}{a}\right) d t, f \in L^{2}(R), a, b \in R, a \neq 0,
$$

where $\Psi$ is the basic wavelet, $a$ characterizes scale, $b$ is the time. During scale $a$ decrease, wavelet transform coefficients $W_{\Psi} f_{b, a}$ characterize function $f$ properties in the vicinity of $t=b$. On small scales $s$ absolute values of the coefficients $W_{\Psi} f_{b, a}$ are small except for the vicinities containing local peculiarities of the function $f$ (Jaffar theorem) [14, 15]. Daubechies 3 wavelet was used in the paper [15]. Using (1) for IMF Bz time series, changes at times $b$ were detected on different scales (periods) $a$. Positive and negative changes in the data were divided on the basis of

$$
W_{\Psi} f_{b, a}=\left\{\begin{array}{l}
\left(W_{\Psi} f_{b, a}\right)^{+}, \text {if } W_{\Psi} f_{b, a}>0 \\
\left(W_{\Psi} f_{b, a}\right)^{-}, \text {if } W_{\Psi} f_{b, a} \leq 0
\end{array}\right.
$$

The obtained changes were described through the introduced intensity of IMF $\mathrm{Bz}$ disturbance for negative values

$$
I_{b}^{-}=\sum_{b}\left(W_{\Psi} f_{b, a}\right)^{-}
$$

To estimate the dependence of AE on IMF Bz data, we used the correlation coefficient

$$
r=\frac{\sum_{i}\left(x_{i}-\bar{x}\right)\left(z_{i}-\bar{z}\right)}{\sqrt{\sum_{i}\left(x_{i}-\bar{x}\right)^{2} \sum_{i}\left(z_{i}-\bar{z}\right)^{2}}},
$$


where $\bar{x}=\frac{1}{n} \sum_{i} x_{i}, \bar{z}=\frac{1}{n} \sum_{i} z_{i}$

Correlation coefficient significance was estimated on the basis of Student's $t$-criterion [16].

We also developed regression models of the type

$$
Y=c_{1} x_{1}+c_{2} x_{2}+\cdots+c_{n} x_{n}+d
$$

where $\mathrm{Y}$ is the total value of AE index over 24 hours, $x_{i}$ are the factors affecting $\mathrm{AE}, c_{i}, d$ are the model estimated parameters.

To estimate the statistical significance of the obtained models, the following quantities were used:

- determination coefficient

$$
R^{2}=1-\frac{\sum_{i}\left(z_{i}-\hat{z}_{i}\right)^{2}}{\sum_{i}\left(z_{i}-\bar{z}_{i}\right)^{2}}
$$

where $\bar{z}=\frac{1}{n} \sum_{i} z_{i}, z_{i}, \hat{z}_{i}$ are the real and calculated values of the interpretable variable; - F-statistics

$$
F=\frac{R^{2} / n}{\left(1-R^{2}\right) /(m-n-1)}
$$

where $m$ is the number of interpretable variables, $n$ is the number of observations;

- Student's distribution to estimate the significance of regression coefficients

$$
t=\frac{B}{S b}
$$

where $B$ is the estimated regression coefficient, $S b$ is the standard error of regression coefficient.

\section{Results}

For the period 01.01.1999 - 28.02.2018, we obtained 167976 pairs of values, corresponding to IMF Bz and AE hourly data. IMF Bz positive values were equaled to zero. In the remaining negative values, IMF Bz southward turn duration was from 1 hour to 69 hours. It is known that southern $\mathrm{Bz}$ duration and its decrease level influence the disturbance degree in the magnetosphere and ionosphere [17, 18]. To estimate the changes occurring in IMF $\mathrm{Bz}$, southern $\mathrm{Bz}$ periods were grouped according to duration (Fig. 1a). Maximum number of periods, when $\mathrm{Bz}$ was negative during an hour, was 7028 (Fig. 1a). That is the most significant group of events. As the period duration increased, the number of events became smaller. For example, the number of events with the duration of 24 hours was 16. Fig. $1 \mathrm{~b}$ shows the total decrease of Bz-component for the periods with different duration

$$
S_{t}=\sum_{p} \sum_{1}^{t} B z_{t}
$$

where $B z_{i}$ is the Bz-component value within $i$-m hour, $t$ is the period duration in hours, $p$ is the number of periods with the duration of $t$ hours. Thus, the greatest decrease was observed during the periods with the duration of 3 hours. Periods with the duration of one hour showed $\mathrm{Bz}$ decrease compared with the periods with the duration of 10 hours. Analysis of Fig. 1 
illustrates that the relation between the IMF Bz southward turn duration and its summary decrease is linear. At the same time, it is generally accepted that the most significant contribution of IMF BZ energy into geospheric shells takes place during magnetic storms and during long $\mathrm{Bz}$ decreases. In these cases, IMF BS southward turn usually lasts for more than 3 hours $[4,5,17-20]$.
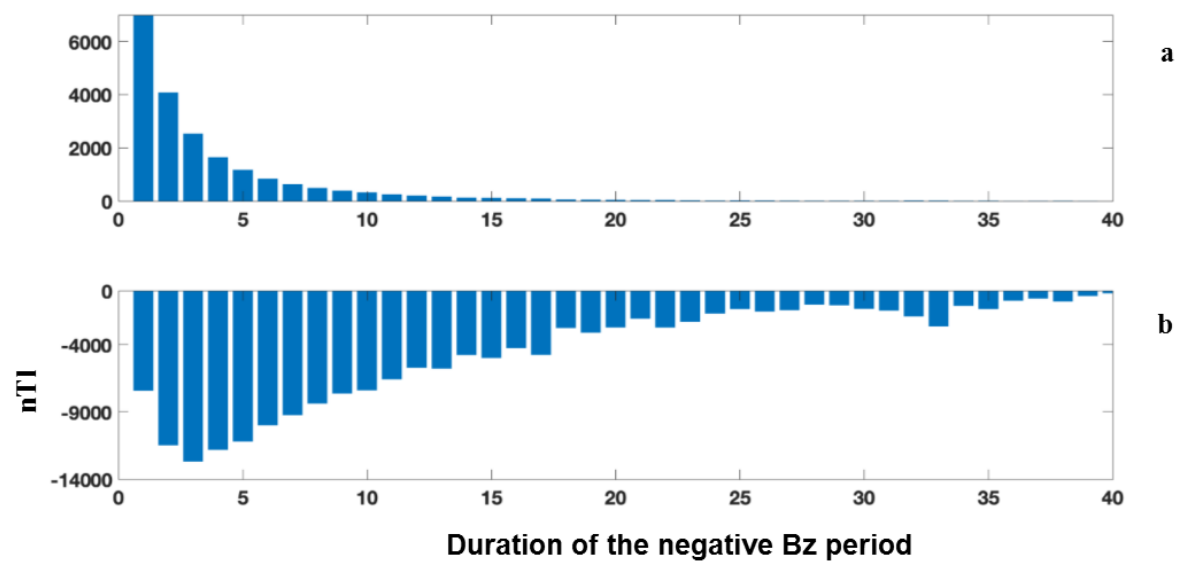

Fig. 1. Distribution of negative IMF BZ according to duration periods. a - number of periods corresponding to duration; $\mathrm{b}$ - summary $\mathrm{Bz}$ for the periods of corresponding duration.

By the means of continuous wavelet transform, negative IMF $\mathrm{Bz}$ was divided into frequency ranges: up to 4 hours, 5-8 hours, 9-16 hours and 17-24 hours. An example of data processing is shown in Fig. 2. During the period illustrated in the figure, there were both calm segments (March 16, 2015), small amplitude fluctuations (March 14, 2015) and significant changes (March 17, 2015) entailing a magnetic storm (Fig. 2 a). The graph of intensity for the period of 1-4 hours shows the changes compared with short-period processes of $\mathrm{Bz}$ change (Fig. 2b). As period duration increased, intensity graphs become smooth and reflect the most extensive Bz fluctuations (Fig. $2 \mathrm{c}-$ e). AE index correlates well with $\mathrm{Bz}$ changes until March 17, 2015. From March 18, AE index had fluctuations occurring almost at the same time with $\mathrm{Bz}$ fluctuations but they had larger amplitude than before the magnetic storm (Fig. 2 f). 

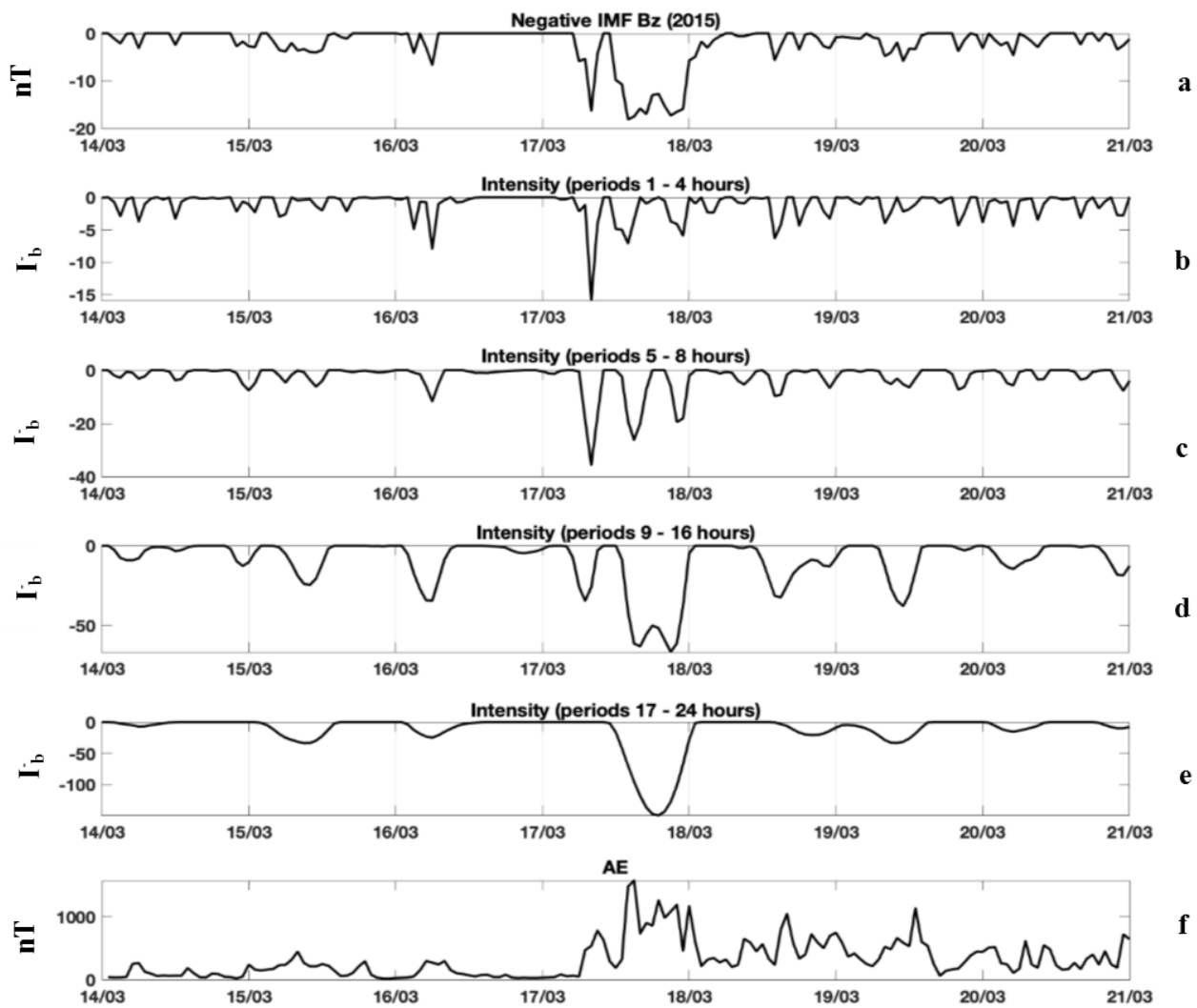

Fig. 2. Processing results of IMF Bz for the period March $14-20,2015$.

After IMF Bz data processing, we obtained 4 time series which represent the intensity values for different frequency ranges. The values for every 24 hours were summed up and correlation coefficients between $\mathrm{Bz}$ intensity and $\mathrm{AE}$ index were found. The obtained correlation coefficients are illustrated in Table 1. Analysis of the data in Table 1 shows linear statistically significant relation between the estimated quantities and confirms the fact of considerable impact of IMF Bz parameters on auroral electrojet state. IMF Bz fluctuations with the period of 1-4 hours make the greatest effect on AE changes. Statistical significance of the coefficients, according to Student's criterion, is quite high.

Table 1. Correlation coefficients of IMF Bz parameters and AE index.

\begin{tabular}{|c|c|c|c|c|c|}
\hline No & $\begin{array}{c}\text { Correlation } \\
\text { coefficient } \\
\mathrm{r}\end{array}$ & T-statistics & $\begin{array}{c}\text { Period } \\
\text { (hours) }\end{array}$ & $\begin{array}{c}\text { IMF Bz } \\
\text { data }\end{array}$ & AE index \\
\hline 1 & 0.66 & -74.73 & $1-4$ & $\begin{array}{c}\text { 24-hour } I_{b}^{-} \\
\text {sum }\end{array}$ & 24-hour sum \\
\hline 2 & 0.61 & -65.92 & $5-8$ & $\begin{array}{c}\text { 24-hour } I_{b}^{-} \\
\text {sum }\end{array}$ & 24-hour sum \\
\hline 3 & 0.58 & 60.37 & $9-16$ & $\begin{array}{c}\text { 24-hour } I_{b}^{-} \\
\text {sum }\end{array}$ & 24-hour sum \\
\hline 4 & 0.56 & -56.86 & $17-24$ & $\begin{array}{c}\text { 24-hour } I_{b}^{-} \\
\text {sum }\end{array}$ & 24-hour sum \\
\hline
\end{tabular}


Based on the obtained results, the following regression models were developed.

1. Model of dual regression:

$$
Y=-89.78 x_{B z}+2102
$$

where $x_{B z}$ is the sum of negative IMF BZ over a day.

The model F-statistics value was $F=7075$. For the given level of significance $\alpha=0,01$ (probability 99\%), that indicates the presence of statistically significant linear relation between IMF $\mathrm{Bz}$ and $\mathrm{AE}$. The t-statistics value $t=-84.11$ exceeds the critical value $t_{c r i t}=3.2$ (for the significance level $\alpha=0.001$ ) that also confirms the statistical significance between the values. Determination coefficient of the model $R^{2}=0.5$ indicates the fact that the model explains about $50 \%$ of values.

2. Model of multiple regression:

$$
Y=-60.29 x_{B z}-116.7 x_{I_{b}^{-}}+1175,
$$

where $x_{B z}$ is the sum of negative IMF Bz over a day, $x_{I_{b}^{-}}$is the sum of $I_{b}^{-}$over 24-hour period for the frequency range of 1-4 hours.

The obtained model F-statistics value $F=4548$, for the given significance level $\alpha=0.001$ (probability $99.9 \%$ ) indicates the presence of statistically significant relation between the explicative variables (IMF Bz and $I_{b}^{-}$for the period of 1-4 hours) and AE value. The obtained t-statistics values, $t_{B z}=-44.2$ and $t_{I_{b}^{-}}=-31.71$ show the statistically significant impact of each parameter on AE value. Determination coefficient of the model $R^{2}=0.56$ exceeded the determination coefficient of the dual regression model (see above) and indicates that the variables included into the model explain about $56 \%$ from the total number of values.

\section{Conclusions}

Based on the analysis of IMF Bz for the period 1999-2018, it was shown that periods with the duration of 3 hours make the greatest contribution into $\mathrm{Bz}$ negative state. The paper confirms the statistically significant relation between $\mathrm{AE}$ parameters and the intensity measure $I_{b}^{-}$introduced for IMF Bz. Estimation of correlation coefficients showed that fluctuations of IMF Bz negative values with the periods of $1-4$ hours make the greatest influence on $\mathrm{AE}$ index. We have developed regression models and determined IMF $\mathrm{Bz}$ parameters having statistically significant impact on energy transfer into auroral electrojet.

The work was carried out according to the Subject AAAA-A17-117080110043-4" «Dynamics of physical processes in the active zones of near space and geospheres». The authors appreciate the help of the Institutes providing the data of interplanetary magnetic field OMNI database (https://omniweb.gsfc.nasa.gov/ow.html), World Data Center for Geomagnetism, Kyoto (http://wdc.kugi.kyoto-u.ac.jp).

\section{References}

1. J. W. Dungey, Phys. Rev. Lett., 6, 47-48 (1961)

2. S. W. H. Cowley, EOS T. Am. Geophys. Un., 76, 525-532 (1995) 
3. B. T. Tsurutani, B. E. Goldstein, E. J. Smith, W. D. Gonzalez, F. Tang, S. I. Akasofu, R. R. Anderson, Planet. Space Sci., 38, 109-126, (1990)

4. A. M. Souza, E. Echer, M. J. A. Bolzan, Ann. Geophys., 36, 205-211 (2018)

5. F. L. Guarnieri, B. T. Tsurutani, L. E. A. Vieira, R. Hajra, E. Echer, A. J. Mannucci, W. D. Gonzalez, Nonlin. Processes Geophys., 25, 67-76 (2018)

6. A. M. Souza, E. Echer, M. J. A. Bolzan, R. Hajra, J. Atmos. Sol.-Terr. Phy., 149, 81-86 (2016)

7. R. Hajra, E. Echer, B. T. Tsurutani, W. D. Gonzalez, J. Geophs. Res., 119, 2675-2690 (2014)

8. M. Sugiura, T. N. Davis, J. Geophys. Res., 71, 785-801 (1966)

9. F. L. Guarnieri, Recurrent Magnetic Storms: Corotating Solar Wind Streams (Am. Geophys. Univ. Press, Washington, DC, 2006)

10. B. T. Tsurutani, W. D. Gonzalez, Planet. Space Sci., 35, 405-412 (1987)

11. R. Hajra, E. Echer, B. T. Tsurutani, W. D. Gonzalez, J. Geophys. Res.-Space, 118, 56265638 (2013)

12. R. J. A. Little, D.B. Rubin, Statistical analysis with missing data (Wiley-Interscience, N.Y., 2002)

13. S. Mallat, A wavelet tour of signal processing (Academic Press, London, 1999)

14. C. K. Chui, An introduction in wavelets (Academic Press, New York, 1992)

15. I. Daubechies, Ten Lectures on wavelets (SIAM, Philadelphia, 1992)

16. Student, Biometrika, 6 (1) (1908)

17. I. Tsagouri, A. Belehaki, J. Space Weather Space Clim., 5, A9 (2015)

18. W. D. Gonzalez, B. T. Tsurutani, Planet. Space Sci., 35, 1101-1109 (1987)

19. E. Tanskanen, J. Slavin, D. Fairfield, D. Sibeck, J. W. Gjerloev, T. Mukai, A. Ieda, T. Nagai, J. Geophys. Res., 110 (2005)

20. B.O. Adebesin, V.U. Chukwuma, Acta Geod. Geoph. Hung 43, 1-15 (2008) 